\title{
THE IMPACT OF COGNITIVE DEFICITS AND SPASTICITY ON DRIVING SIMULATOR PERFORMANCE IN MULTIPLE SCLEROSIS
}

\author{
Thomas D. Marcotte ${ }^{1}$, Theodore J. Rosenthal ${ }^{2}$, Jody Corey-Bloom ${ }^{1}$, \\ Erica Roberts ${ }^{1}$, Sara Lampinen ${ }^{1}$, and R. Wade Allen ${ }^{2}$ \\ ${ }^{1}$ University of California \\ San Diego, California, USA \\ ${ }^{2}$ Systems Technology, Inc. \\ Hawthorne, California, USA \\ E-mail: tmarcotte@ucsd.edu
}

\begin{abstract}
Summary: Multiple sclerosis (MS) is a demyelinating disease that can result in numerous sequelae. Although spasticity and cognitive dysfunction are common in MS, few studies have examined the impact of both factors on driving abilities in persons with physical impairments. The present study assessed driving performance in control participants and MS patients with documented spasticity using two brief simulations designed to measure lane tracking (under high cognitive load) and car following behavior. Seventeen MS patients and 9 controls participated in the study. The MS cohort exhibited a broad range of cognitive functioning (normal to significant impairment) and disability (Expanded Disability Status Scale scores of 3.0 to 7.5). Eight of the MS patients had significant spasticity in their right knee based upon the Modified Ashworth Spasticity Scale. MS patients had greater difficulty than controls on the simulations, particularly on the car following task. MS participants also tended to drive at higher speeds than the control participants. Within the MS cohort, cognitive dysfunction was most strongly associated with lane tracking decrements, whereas the possible relationship between cognitive function and car following behavior was eclipsed by lower limb spasticity. Spastic individuals had greater difficulty mirroring speed changes in the lead car, and were approximately one second slower in responding to its accelerations and decelerations. The current simulations provide important data regarding the impact various MS sequelae may have on driving performance, and may ultimately lead to clinical recommendations regarding specific driving behaviors and their associated risks.
\end{abstract}

\section{INTRODUCTION}

Multiple sclerosis (MS) is a chronic inflammatory, progressive demyelinating disease that causes disability in middle-aged adults. The sequelae from MS vary from patient to patient; the most common manifestations including spasticity, visual blurring, ataxia, and cognitive impairment. Spasticity is an abnormal increase in muscle tone that is typically velocity-dependent (how fast the joint is moved through its range), and frequently results in disability.

Such sequelae often affect everyday functioning, including the patient's ability to safely drive an automobile. For example, MS patients were found to have a higher rate of automobile crashes than controls (Lings, 2002; Schultheis, Garay, Millis, \& Deluca, 2002), and to have greater difficulty on a driving simulator (Kotterba et al., 2003). Schultheis et al. (2001) reported that MS participants with documented cognitive impairment performed more poorly on measures 
associated with driving performance, including the Neurocognitive Driving Test (NDT) and the Useful Field of View (Ball \& Roenker, 1998), a test of visual attention that has been shown to be predictive of driving accidents in older adults (Owsley et al., 1998).

In the interest of addressing the potential impact of cognitive impairment on driving, many studies have opted to exclude MS participants with greater than minimal physical disability. This potentially excludes an important, and large, subgroup of MS patients.

The goal of the present study was to examine the contribution of both physical and cognitive sequelae to driving impairment in drivers with documented MS-associated spasticity. To assess different components of driving performance, we utilized simulations that measured (1) lane tracking under high cognitive workload, and (2) response to lead-car speed changes, emphasizing lower limb mobility under limited cognitive load.

\section{METHODS}

\section{Participants}

Participants consisted of 17 persons with documented multiple sclerosis (MS) and 9 controls. The MS participants were enrolled in a clinical trial for the treatment of MS-related spasticity, and only pre-treatment, baseline data were used in these analyses. In order to be included in this study, all participants had to have routinely driven an automobile in their lifetime, including a minimum of 250 miles in the past year. MS participants were included if they had: (1) definite or probable MS, (2) complaints of spasticity and at least moderate increase in tone, (3) were between the ages of 18 and 65, (4) fluent in English, and (5) on a stable dose of either Baclofen or Zanaflex for at least three months. Participants were excluded if they had: (1) significant Axis I psychiatric disorder or neurological disease other than MS, (2) recent active substance abuse, (3) an unstable medical problem, (4) required benzodiazepines to control spasticity, or (5) used sedative or high doses of analgesic medications frequently. Control participants were recruited from the community, required to be current drivers, and were excluded if there was a history of any CNS disorder that might affect cognition, evidence of a head injury with loss of consciousness $>30$ minutes, or a history of drug abuse.

The two groups were well matched with respect to age, education, ethnicity and driving history, although there were slightly more females in the MS group (Table 1).

Table 1. Demographics of the control and MS participants

\begin{tabular}{lccc}
\hline & $\begin{array}{c}\text { Control } \\
(\mathbf{n}=\mathbf{9})\end{array}$ & $\begin{array}{c}\text { MS } \\
(\mathbf{n}=\mathbf{1 7})\end{array}$ & $\boldsymbol{p}$ \\
\hline Age & $48.3(14.0)$ & $49.6(7.9)$ & .78 \\
Education (years) & $15.3(2.4)$ & $14.5(2.1)$ & 39 \\
Gender (\% female) & $33 \%$ & $65 \%$ & .22 \\
Ethnicity (\% white) & $89 \%$ & $94 \%$ & .89 \\
Miles driven in past year & $6,345(3,346)$ & $4,724(3,573)$ & .27 \\
\hline
\end{tabular}


The MS group evidenced a range of disability as assessed by the Kurtzke Expanded Disability Status Scale (EDSS). The median score for the group was 6.0 (intermittent or unilateral constant assistance to walk about 100 meters; EDDS range $=3.0$ to 7.5). On the Ashworth Spasticity Scale (Bohannon \& Smith, 1987, see below), 8/17 (47\%) of the cohort had clinically significant spasticity in their right knee, while $9 / 17$ (53\%) did not.

\section{Procedures}

Neuropsychological testing. MS participants completed a brief neuropsychological test battery designed to assess domains frequently affected by multiple sclerosis. The battery assessed Motor functioning (Grooved Pegboard Test), Speed of Information Processing (Trailmaking Test Part A, WAIS-III Digit Symbol), Attention/Working Memory (Paced Auditory Serial Addition Test), Executive Functioning (Trailmaking Test Part B), and Learning and Memory (Hopkins Verbal Learning Test). Control subjects completed a subset of these tests (Trailmaking Test, Digit Symbol, Hopkins Verbal Learning Test) to ensure that there were no significant, undiagnosed disorders. Overall cognitive functioning was estimated using the Global Deficit Score (GDS) approach, in which impaired performances on cognitive tests (based upon demographicallycorrected $\mathrm{T}$ scores) are weighted more heavily than average and above average performance. Test performance in the normal range yields a GDS of 0 , whereas severe impairment would be assigned a score of 5 (i.e., a higher GDS indicates greater impairment). This approach has been shown to be sensitive to cognitive dysfunction for a variety of disorders (Carey et al., 2004).

Spasticity assessment. MS participants were assessed for level of spasticity using the Modified Ashworth Spasticity Scale (Bohannon \& Smith, 1987), an examiner-administered, independent measure of spasticity. Spasticity was rated bilaterally for the elbow, hip and knee. Since the focus of this study was performance on the driving simulator, in particular the effect that spasticity might have on pedal performance, we used the participant's right knee as the primary joint of interest.

Driving simulation. All participants completed a 15-minute driving simulation. The simulation was presented on a Pentium III PC computer using a 17" monitor at $1280 \times 1024$ resolution, and running STISIM Drive version 2.0 software (Systems Technology, Inc., Hawthorne, CA). Hardware included a steering wheel, turn signal, and brake/accelerator pedals (Rosenthal, 1999). The system provided auditory feedback (e.g., engine noise). Two separate tasks were embedded within the one simulation. The first simulation task, lasting approximately 7 minutes, required participants to maintain lane position while holding a constant speed (55 mph). Cognitive workload, and thus the sensitivity of the measure, was increased by having participants attend to divided attention tasks in the corner of the monitor; they were to respond to a triangle in the left or right corners of the monitor by pressing the left or right turn signal, respectively, and to a picture of a horn in either corner by pressing a button on the console. The scenery consisted of a straight, two-lane roadway with mountains and clouds on the horizon. During the simulation subjects were not required to negotiate any curves or turns, and there were no traffic, pedestrians, or traffic control devices used during the drive. The primary outcome was the standard deviation of lateral position (SDLP), a measure of how well participants maintain their lane position. This measure has been shown to have good test-retest reliability (Marcotte et al., 2005), and previous studies have shown performance on versions of this simulator to be sensitive to illicit substance 
use (Stein, Allen, Cook, \& Karl), sleep apnea (Risser, Ware, \& Freeman, 2000), and HIVassociated cognitive impairment (Marcotte et al., 1999).

The second simulation lasted approximately 8 minutes and required participants to follow a lead vehicle at a safe and constant distance. The lead vehicle changed speed following a sinusoidal wave with an amplitude of $6 \mathrm{mph}$. Each change in speed occurred over a period of 1 minute. The mean velocity of the lead vehicle was $55 \mathrm{mph}$. The primary outcomes were the coherence (correlation coefficient) between the participant and lead car (a general measure of the participants ability to accurately track the speed variations of the lead car), time delay (or the reaction time to changes in the lead car's speed), and modulus (amplitude ratio; the degree to which participants under- or over-shoot the distance with the lead car). Details of this simulation and outcome measures can be found in the paper presented by Allen et al. (2005) at this conference.

Statistical analyses. A number of the driving simulation variables were not normally distributed, and thus were transformed. SDLP, modulus, and Time Delay were log transformed, while Coherence was transformed using the Box Cox procedure. Raw scores are shown in tables and figures to aid interpretation; all statistical analyses utilized the transformed variables. Since the sample sizes for this study are small, yielding limited power, we report Cohen's $d$, an indicator of the effect size of the differences between two groups. Following Cohen's recommendations (1988), effect sizes of .2 were considered "small," .5 "medium," and .8 "large."

\section{RESULTS}

The initial analyses involved a comparison of the MS and control participants on each of the driving simulator outcomes. There was a large effect size for average speed, with the MS patients driving significantly faster on the simulation, and a medium effect size for SDLP. Speed deviation did not differ by group. MS participants had greater difficulty than controls in tracking the movements of the lead car on the car following task (coherence correlation of .61 vs. .83; large effect size), and MS participants were more slow to respond to the changes in the lead car's speed (Time Delay; large effect size). The MS participants were no more likely to over- or under-shoot the distance between the lead car. The modulus and time delay findings were similar when two participants with a coherence of less than .3 (indicating inconsistent/incorrect performance on the task) were removed from the analyses.

Table 2. Simulator performance of the control and MS participants

\begin{tabular}{lcccc}
\hline & $\begin{array}{c}\text { Control } \\
(\mathbf{n}=\mathbf{9})\end{array}$ & $\begin{array}{c}\text { MS } \\
(\mathbf{n}=\mathbf{1 7})\end{array}$ & $\begin{array}{c}\text { Cohen's } \\
\boldsymbol{d}\end{array}$ & $\boldsymbol{p}$ \\
\hline Lane Tracking & & & & \\
$\quad$ Average Speed & $55.2(7.0)$ & $61.8(8.5)$ & .87 & .06 \\
Average Speed Deviation & $3.3(2.5)$ & $3.4(1.8)$ & .05 & .86 \\
$\quad$ SDLP (log) & $.38(.07)$ & $.42(.11)$ & .43 & .24 \\
Car Following & & & & \\
$\quad$ Coherence & $.83(.12)$ & $.61(.22)$ & 1.2 & .02 \\
Time Delay (sec) & $.39(.28)$ & $1.9(2.0)$ & 1.1 & .04 \\
$\quad$ Modulus & $1.1(.11)$ & $1.2(.15)$ & .12 & .70 \\
\hline
\end{tabular}


We next sought to determine whether cognitive functioning and/or spasticity indicators were predictors of simulator performance within the MS group. Analyses included (a) correlational analyses using the continuous global deficit score as an indicator of neurocognitive status, and (b) clinically significant vs. insignificant spasticity in the right knee (Ashworth $\geq 2$ ) as the indicator of task relevant spasticity.

Greater cognitive dysfunction was associated with faster driving speed $(\mathrm{r}=.51, p=.008)$, greater variability in speed maintenance $(\mathrm{r}=.39, p=.047)$, and a larger SDLP $(\mathrm{r}=.65, p=.005)$. Neuropsychological functioning was not predictive of performance on the car following task: coherence $(\mathrm{r}=-0.30, p=.24)$, modulus $(\mathrm{r}=.15, p=.58)$ or Time Delay $(\mathrm{r}=.33, p=.21)$.

Conversely, as seen in Figure 1, spasticity was significantly associated with a number of car following outcomes, but not SDLP. The largest effect size was for coherence (Cohen's $d=1.0$, $p=.047)$, followed by the Time Delay $(d=.62, p=.25)$. Small to medium effect sizes were found for Modulus $(d=.40)$ and $\operatorname{SDLP}(d=.36)$.
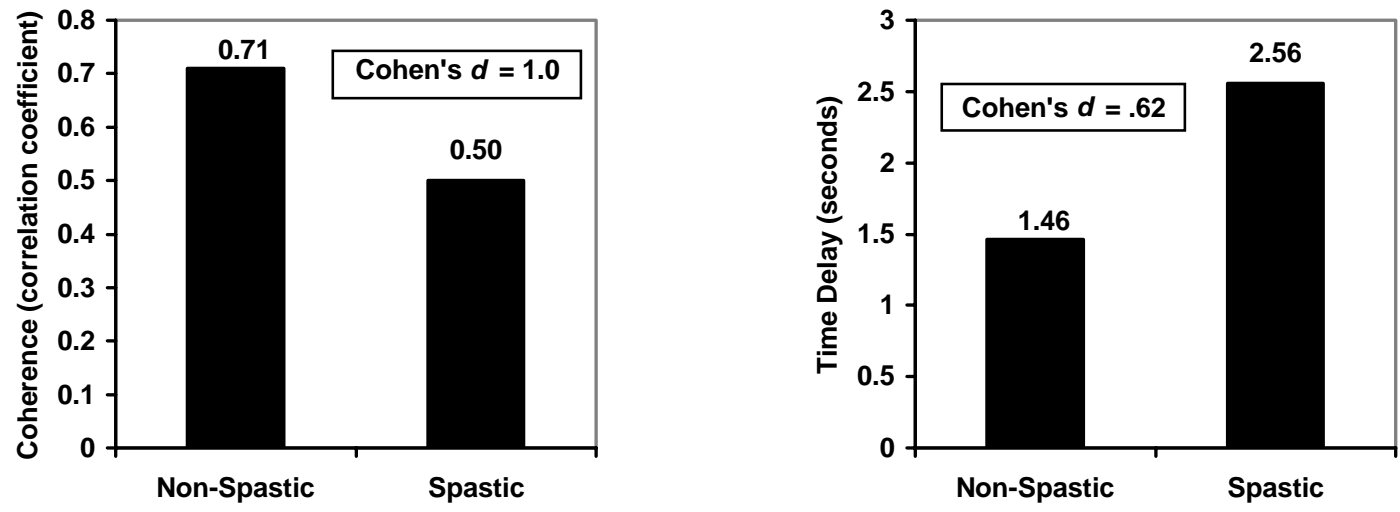

Figure 1. Performance on the car following task (MS patients only)

\section{DISCUSSION}

The current study is one of few to address driving performance in MS drivers with significant spasticity. Using two simulator paradigms, MS participants exhibited decreased driving skills relative to control participants, particularly with respect to their ability to adjust to changes in the speed of a lead car (making adjustments, on average, approximately 1 second slower than controls).

Within the MS cohort, cognitive and physical impairments independently contributed to specific deficits in driving performance. Cognitively impaired participants had greater difficulty on a simulation requiring lane tracking under high cognitive load, suggesting that these individuals may have difficulty under complex driving situations. Cognition was not significantly associated with poor performance on the car following task, however. Success on this measure, rather, was more impacted by lower limb spasticity, with the spastic group having greater trouble in adjusting to changes in lead car speed, presumably due to less muscle control in the area of the accelerator pedal. In other studies, reduced car following behavior has been associated with alcohol and 
marijuana administration (Ramaekers, Robbe, \& O'Hanlon, 2000) and prescription drug administration (Weiler et al., 2000). We suspect that in the present study, an underlying relationship between car following and cognitive status was overshadowed by the impact of lower limb spasticity.

Interestingly, the MS patients in the current study tended to drive at higher speeds on the simulator, which is somewhat concerning considering the delay in responding to lead car accelerations and decelerations.

Limitations to the present study include the small sample sizes, and limited simulator evaluations. The simulations were intentionally brief in order to be practical for use in clinical trials, but there is likely a cost with respect to sensitivity and reliability of the findings. There are also many other sequelae associated with MS that may impact driving performance, and these were not addressed here.

Nonetheless, the present study does provide new data regarding the impact that various MS conditions may have on a person's ability to safely drive an automobile. Clinicians may want to consider discussing the potential driving conditions (e.g., close car following, multi-tasking) that may put particular patients at risk for automobile accidents.

\section{ACKNOWLEDGMENTS}

This study was supported by the University of California Center for Medicinal Cannabis (C00-SD102, T.D. Marcotte, PI; C00-SD-103, J. Corey-Bloom, PI) and the National Institute of Mental Health (R42 MH57593, T.D. Marcotte, PI).

\section{REFERENCES}

Allen, R. W., Marcotte, T. D., Rosenthal, T. J., \& Aponso, B. L. (2005). Driver assessment with measures of continuous control behavior. Paper presented at the 3rd International Driving Symposium on Human Factors in Driver Assessment, Training, and Vehicle Design, Rockport, ME.

Ball, K., \& Roenker, D. (1998). Useful Field of View. San Antonio: The Psychological Corporation.

Bohannon, R. W., \& Smith, M. B. (1987). Interrater reliability of a modified Ashworth scale of muscle spasticity. Physical Therapy, 67(2), 206-207.

Carey, C. L., Woods, S. P., Gonzalez, R., Conover, E., Marcotte, T. D., Grant, I., \& Heaton, R. K. (2004). Predictive validity of global deficit scores in detecting neuropsychological impairment in HIV infection. Journal of Clinical and Experimental Neuropsychology, 26(3), 307-319.

Cohen, J. (1988). Statistical Power Analysis for the Behavioral Sciences (2nd ed.). Hillsdale, New Jersey: Lawrence Erlbaum Associates.

Kottera. S., Orth, M., Eren, Fangerau, T., \& Sindern, E. (2003). Assessment of driving performance in patients with relapsing-remitting multiple sclerosis by a driving simulator. European Neurology, 50(3), 160-164. 
Lings, S. (2002). Driving accident frequency increased in patients with multiple sclerosis. Acta Neurologica Scandinavica, 105(3), 169-173.

Marcotte, T. D., Heaton, R. K., Wolfson, T., Taylor, M. J., Alhassoon, O., Arfaa, K., Ellis, R. J., Grant, I., \& the HNRC Group. (1999). The impact of HIV-related neuropsychological dysfunction on driving behavior. Journal of the International Neuropsychological Society, 5(7), 579-592.

Marcotte, T. D., Scott, J. C., Lazaretto, D., Roberts, E., Bentley, H., Rosenthal, T. J., \& the HNRC Group. (2005). Long-term stability of standard deviation of lateral position in neurocognitively normal and impaired individuals. Advances in Transportation Studies: An International Journal, 57-65.

Owsley, C., Ball, K., McGwin, G., Jr., Sloane, M. E., Roenker, D. L., White, M., \& Overley, T. (1998). Visual processing impairment and risk of motor vehicle crash among older adults. JAMA, 279(14), 1083-1088.

Ramaekers, J. G., Robbe, H. W., \& O'Hanlon, J. F. (2000). Marijuana, alcohol and actual driving performance. Human Psychopharmacology-Clinical and Experimental, 15(7), 551-558.

Risser, M. R., Ware, J. C., \& Freeman, F. G. (2000). Driving simulation with EEG monitoring in normal and obstructive sleep apnea patients. Sleep, 23(3), 393-398.

Rosenthal, T. J. (1999). STISIM Drive User's Manual. Hawthorne, CA: Systems Technology, Inc.

Schultheis, M. T., Garay, E., \& DeLuca, J. (2001). The influence of cognitive impairment on driving performance in multiple sclerosis. Neurology, 56(8), 1089-1094.

Schultheis, M. T., Garay, E., Millis, S. R., \& Deluca, J. (2002). Motor vehicle crashes and violations among drivers with multiple sclerosis. Archives of Physical Medicine and Rehabilitation, 83(8), 1175-1178.

Stein, A. C., Allen, R. W., Cook, M. L., \& Karl, R. L. A simulator study of the combined effects of alcohol and marijuana on driving behavior (DOT HS-806-405): NTIS.

Weiler, J. M., Bloomfield, J. R., Woodworth, G. G., Grant, A. R., Layton, T. A., Brown, T. L., McKenzie, D. R., Baker, T. W., \& Watson, G. S. (2000). Effects of fexofenadine, diphenhydramine, and alcohol on driving performance: A randomized placebo-controlled trial in the Iowa Driving Simulator. Annals of Internal Medicine, 132, 354-363. 